\title{
Low-frequency study of two clusters of galaxies: A2744 and A2219
}

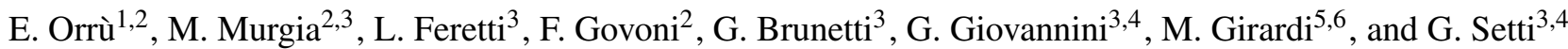 \\ 1 Dipartimento di Fisica, Università degli Studi di Cagliari, Cittadella Universitaria, 09042 Monserrato (CA), Italy \\ e-mail: eorru_s@ira.inaf.it \\ 2 INAF-Osservatorio Astronomico di Cagliari, Loc. Poggio dei Pini, Strada 54, 09012 Capoterra (CA), Italy \\ INAF - Istituto di Radioastronomia, via Gobetti 101, 40129 Bologna, Italy \\ 4 Dipartimento di Astronomia Università degli Studi di Bologna, via Ranzani 1, 40127 Bologna, Italy \\ 5 Dipartimento di Astronomia, Università degli Studi di Trieste, via Tiepolo 11, 34131 Trieste, Italy \\ ${ }^{6}$ INAF-Osservatorio Astronomico di Trieste, via Tiepolo 11, 34131 Trieste, Italy
}

Received 26 July 2006 / Accepted 31 January 2007

\section{ABSTRACT}

\begin{abstract}
Aims. Spectral index images can be used to constraint the energy spectrum of relativistic electrons and magnetic field distribution in radio halos and relics, providing useful information to understand their formation, evolution and connection to cluster merger processes.

Methods. We present low-frequency images of the two clusters of galaxies: A2744 and A2219, in which a wide diffuse emission is detected. Observations were made with the Very Large Array at the frequency of $325 \mathrm{MHz}$. For both clusters deep Very Large Array $1.4 \mathrm{GHz}$ observations are available. Combining the $325 \mathrm{MHz}$ and $1.4 \mathrm{GHz}$ data, we obtained the spectral index images and the brightness radial profiles of the diffuse radio emission with a resolution of $\sim 1^{\prime}$.

Results. The azimuthally averaged spectral index in A2744 is constant to a value close to $\alpha \simeq 1$ up to a distance of $1 \mathrm{Mpc}$ from the cluster center. However, the spectral index image shows the presence of localized regions in which the radio spectrum is significantly different from the average. The observed spectral index variations range from a minimum of $\alpha \simeq 0.7 \pm 0.1$ to a maximum $\alpha \simeq 1.5 \pm 0.2$. From the comparison of the spectral index with the X-ray data it is found for the first time that the flat spectrum regions of the radio halo tend to have higher temperature. In the case of A2219, the radio emission in the central regions of the cluster is dominated by a blend of discrete sources. The azimuthally averaged radio spectrum is $\alpha \simeq 0.8$ in the central region of the cluster and is close to $\alpha \simeq 1$ in the radio halo. The limited sensitivity of the $325 \mathrm{MHz}$ image does not allow us to detect all the radio halo structure seen at $1.4 \mathrm{GHz}$ and therefore no constraints on the point-to-point variations of the spectral index have been obtained for this cluster.
\end{abstract}

Key words. galaxies: clusters: individual: A2744 - galaxies: clusters: individual: A2219 - galaxies: intergalactic medium

\section{Introduction}

In the hierarchical merging scenario, large-scale-structures form as the result of several merger events (e.g. Evrard \& Gioia 2002). Clusters of galaxies are the most massive gravitationally bound objects in the Universe and they are structures still forming at the present epoch.

The presence of wide diffuse radio sources associated with the intra-cluster medium (ICM) has been detected in an increasing number of massive, merging clusters of galaxies. These synchrotron diffuse radio sources are characterized by a typical size of about $1 \mathrm{Mpc}$, low surface brightness $\left(\simeq 10^{-6} \mathrm{Jy} / \operatorname{arcsec}^{2}\right.$ at $1.4 \mathrm{GHz})$ and steep radio spectrum ${ }^{1}(\alpha \geq 1$.). They are classified as halos if they are located in the cluster center or relics, if they are in the peripheral regions of the cluster (e.g. Giovannini $\&$ Feretti 2002). Radio halos and relics demonstrate the existence of relativistic electrons and large scale magnetic fields in the ICM. Much effort has been made to explain the origin of halos and relics. The relic emission is believed to be caused by the propagation of shock waves produced during cluster merger events. In this case, the radio emission traces the rim of the shock wave in which electrons are injected and/or re-accelerated (e.g. Rottgering et al. 1997; Enßlin et al. 1998). In the case

\footnotetext{
${ }^{1} S(v) \propto v^{-\alpha}$
}

of radio halos, it is required that either the electrons are reaccelerated (primary models: e.g. Tribble 1993; Brunetti et al. 2001; Petrosian 2001; Brunetti et al. 2004; Fujita et al. 2003; Cassano \& Brunetti 2005) or continuously injected over the entire cluster volume by hadronic collisions (secondary models: e.g. Dennison 1980; Blasi \& Colafrancesco 1999; Dolag \& Enßlin 2000). On the other hand, the strength and structure of the magnetic fields have been estimated and simulated with different methods (see e.g. Carilli \& Taylor 2002; and Govoni \& Feretti 2004 , for reviews). Spectral index maps of radio halos and relics are promising tools in the understanding of their formation, evolution and connection to cluster merger processes. Moreover they provide important information on the energy spectrum of relativistic electrons and magnetic field distribution (Brunetti et al. 2001; Feretti et al. 2004).

Given the large angular sizes $\left(\sim 10^{\prime}\right)$ and steep radio spectrum of halos and relics, suitable spectral index images can only be obtained at frequencies lower than $\sim 1 \mathrm{GHz}$. Nowadays Coma (Giovannini et al. 1993), A2163, A665 (Feretti et al. 2004) and A3562 (Giacintucci et al. 2005) are the only radio halos for which spectral index maps were presented in the literature. In line with primary models, in all these clusters the spectral index maps reveal patchy structure with, in some cases, a trend 
Table 1. Clusters properties.

\begin{tabular}{ccccc}
\hline \hline Name & $\begin{array}{c}\alpha(\mathrm{J} 2000) \\
(\mathrm{h} \mathrm{m} \mathrm{s})\end{array}$ & $\begin{array}{c}\delta(\mathrm{J} 2000) \\
\left({ }^{\circ}{ }^{\prime \prime \prime}\right)\end{array}$ & $z$ & $\mathrm{kpc} /{ }^{\prime \prime}$ \\
\hline A2744 & $00: 14: 19$ & $-30: 22: 19$ & 0.3080 & 4.502 \\
A2219 & $16: 40: 21$ & $+46: 41: 16$ & 0.2256 & 3.587 \\
\hline
\end{tabular}

Column 1: cluster name; Cols 2: and 3: cluster coordinates from NASA/IPAC extragalactic database (NED); Col. 4: redshift (Struble \& Rood 1999); Col. 5: arcsec to kpc conversion.

showing a progressive steepening of the spectrum with increasing distance from the cluster center to the edge.

In this paper we present $325 \mathrm{MHz}$ images of two clusters: Abell 2744 which presents a central radio halo and a peripheral relic and Abell 2219 which hosts a radio halo. The general characteristics of A2744 and A2219 are listed in Table 1.

The paper is organized as follows. In Sect. 2 we discuss the details about the radio observations and the data reduction. In Sect. 3 we present the radio properties of A2744, we show data at $325 \mathrm{MHz}$, the spectral index map and profile obtained by combining the image at $325 \mathrm{MHz}$ with available data at $1.4 \mathrm{GHz}$. Moreover we compare the spectral index map with the cluster properties in the X-ray and optical wavelength. In Sect. 4 the radio properties of A2219 and the new image at $325 \mathrm{MHz}$ are presented and the spectral index distribution obtained between $325 \mathrm{MHz}$ and $1.4 \mathrm{GHz}$ is shown and discussed. The spectral index behavior in A2744 is analyzed in the framework of a particle re-acceleration model in Sect. 5. Finally, in Sect. 6 we summarize the results of this paper.

Throughout this paper we adopt: $H_{0}=71 \mathrm{~km} \mathrm{~s}^{-1} \mathrm{Mpc}^{-1}$, $\Omega_{\Lambda}=0.73$ and $\Omega_{\mathrm{m}}=0.27$.

\section{Observations and data reduction}

Observations were conducted in the $327 \mathrm{MHz}$ band with the Very Large Array $^{2}$ in different configurations. Observational parameters are summarized in Table 2.

A main problem in low-frequency observations are Radio Frequency Interferences (RFI) that corrupt the data. Particularly in the $327 \mathrm{MHz}$ band, the internal electronic of the VLA gives rise to harmonics that are multiples of 5 and $12.5 \mathrm{MHz}$. To avoid this problem, a bandwidth of $3.125 \mathrm{MHz}$ is used; since it is narrow it can be placed between the location characterized by the so called "comb" of RFI (Kassim et al. 1993). The observations are usually made in spectral line mode, dividing the bandwidth in several channels. This also reduces the bandwidth smearing, which is very strong at low frequency.

Data were calibrated and reduced with Astronomical Image Processing System (AIPS). We performed the amplitude and bandpass calibration with the sources $3 \mathrm{C} 48$ and 3C286 respectively for A2744 and A2219. We used the sources 0025-260 and $1710+460$ for the initial phase calibration for A2744 and A2219, respectively.

A careful data editing has been made in order to excise RFI channel by channel. At the end of this procedure $16 \%$ of data were flagged in A2744. The data-set of the 2004-oct- 4 in BnA configuration was too noisy and it is not presented here. For A2219 flagged data were $10 \%$.

${ }^{2}$ The Very Large Array (VLA) is a facility of the National Radio Astronomy Observatory (NRAO). The NRAO is a facility of the National Science Foundation, operated under cooperative agreement by Associated Universities, Inc.
In the final imaging, for A2744 the data were averaged to 7 channels $390 \mathrm{kHz}$ large, while for A2219 they were averaged to 6 channels $488 \mathrm{kHz}$ large. The data were mapped using a widefield imaging technique, which corrects for distortions in the image caused by the non-coplanarity of the VLA over a wide field of view. A set of small overlapping maps was used to cover the central area of about $\sim 2^{\circ}$ in radius (Cornwell \& Perley 1992). However, at this frequency confusion lobes of sources far from the center of the field are still present. Thus, we also obtained images of strong sources in an area of about $\sim 60^{\circ}$ in radius, searched in the NRAO VLA Sky Survey (NVSS, Condon et al. 1998) catalog. All these "facets" were included in CLEAN and used for several loops of phase self-calibration (Perley 1999). The central frequency of the final images is $325 \mathrm{MHz}$ for both A2744 and A2219.

We corrected the final image for the primary beam effect. For A2744 the achieved rms sensitivity is $0.9 \mathrm{mJy} /$ beam in BnA configuration and $2.3 \mathrm{mJy} /$ beam in $\mathrm{CnB}$ configuration. To improve the $\mathrm{u}-\mathrm{v}$ coverage and sensitivity we combined the $\mathrm{BnA}$ and $\mathrm{CnB}$ data sets. The resulting image has a noise level of $1 \mathrm{mJy} / \mathrm{beam}$. For A2219 the achieved sensitivity is $1.7 \mathrm{mJy} / \mathrm{beam}$ in both arrays. All these values are somewhat higher than the expected thermal noise levels, because of the contribution of several factors: confusion, broad-band RFI, VLA generated RFI and some others still unknown.

\section{Properties of the cluster A2744}

A2744 hosts a central radio halo and a peripheral relic, detected in the NVSS by Giovannini et al. (1999) and confirmed in a deeper observation at $1.4 \mathrm{GHz}$ by Govoni et al. (2001a). The normalized radio and X-ray brightness profiles of the cluster appear to be very similar (Govoni et al. 2001), indicating that there could be an energetic relation between the X-ray thermal emitting gas and the relativistic radio emitting particles.

Girardi \& Mezzetti (2001) and Boschin et al. (2006) derived that galaxies are described by a non-Gaussian velocity distribution. They found two galaxy groups with a mass ratio of 3:1 which are separated by a line-of-sight velocity of $\Delta V \sim 4000 \mathrm{~km} \mathrm{~s}^{-1}$. The main one, the low-velocity group, has a velocity dispersion of $\sigma_{V} \simeq 1200-1300 \mathrm{~km} \mathrm{~s}^{-1}$. The secondary one, the high-velocity group, has a velocity dispersion of $\sigma_{V} \simeq 500-800 \mathrm{~km} \mathrm{~s}^{-1}$.

Another indication that the cluster is out of the equilibrium is given by Allen (1998) who found high discrepancies between $\mathrm{X}$-ray masses and lenses masses.

High resolution Chandra X-ray observations confirm the highly disturbed state of the cluster; temperature and brightness gradients have been measured by Kempner \& David (2004). They found a main merger in the proximity of the cluster center and a smaller one in the North-Western region, where the presence of a sub-cluster is evident. The peak of the radio halo is located near the cluster X-ray center but the radio halo emission is spread up to the North-Western sub-cluster.

Unfortunately the field of view of Chandra does not include the region where the relic is located.

\subsection{Low frequency image of $A 2744$}

The BnA and $\mathrm{CnB}$ configuration images at $325 \mathrm{MHz}$ of A2744 are shown in Fig. 1. In the BnA image, which has a resolution of $21^{\prime \prime} \times 11^{\prime \prime}$, we detected both the halo and the relic. This confirms that the emission of the halo and the relic is not due to the blend 
Table 2. Summary of radio observations.

\begin{tabular}{cccccccc}
\hline \hline Name & $\begin{array}{c}\alpha(\mathrm{J} 2000) \\
(\mathrm{h} \mathrm{m} \mathrm{s})\end{array}$ & $\begin{array}{c}\delta(\mathrm{J} 2000) \\
\left({ }^{\circ},{ }^{\prime \prime}\right)\end{array}$ & $\begin{array}{c}v \\
\mathrm{MHz}\end{array}$ & $\begin{array}{c}\Delta v \\
\mathrm{MHz}\end{array}$ & Configuration & Date & $\begin{array}{c}\text { Duration } \\
\mathrm{h}\end{array}$ \\
\hline $\mathrm{A} 2744$ & 001415 & -302260 & $321.5,327.5$ & 3.125 & $\mathrm{BnA}$ & $2004-$ Oct.-04 & 2.5 \\
& & & $321.5,327.5$ & 3.125 & $\mathrm{BnA}$ & $2004-$ Oct.-07 & 3.5 \\
& & & $321.5,327.5$ & 3.125 & $\mathrm{CnB}$ & $2004-F e b .-06$ & 3.5 \\
$\mathrm{~A} 2219$ & \multirow{2}{*}{164015} & \multirow{2}{*}{+464160} & $321.5,327.5$ & 3.125 & $\mathrm{CnB}$ & 2004-Feb.-12 & 3.5 \\
& & & $321.5,327.5$ & 3.125 & $\mathrm{~B}$ & 2003-Oct.-18 & 2.5 \\
& & & 327.5 & 3.125 & $\mathrm{C}$ & $2004-$ May-15 & 7.5 \\
\hline
\end{tabular}

Column 1: cluster name; Cols. 2, 3: radio pointing position; Col. 4: observing frequency; Col. 5: bandwidth; Col. 6: VLA configuration; Col. 7: date of observations; Col. 8: time of integration.
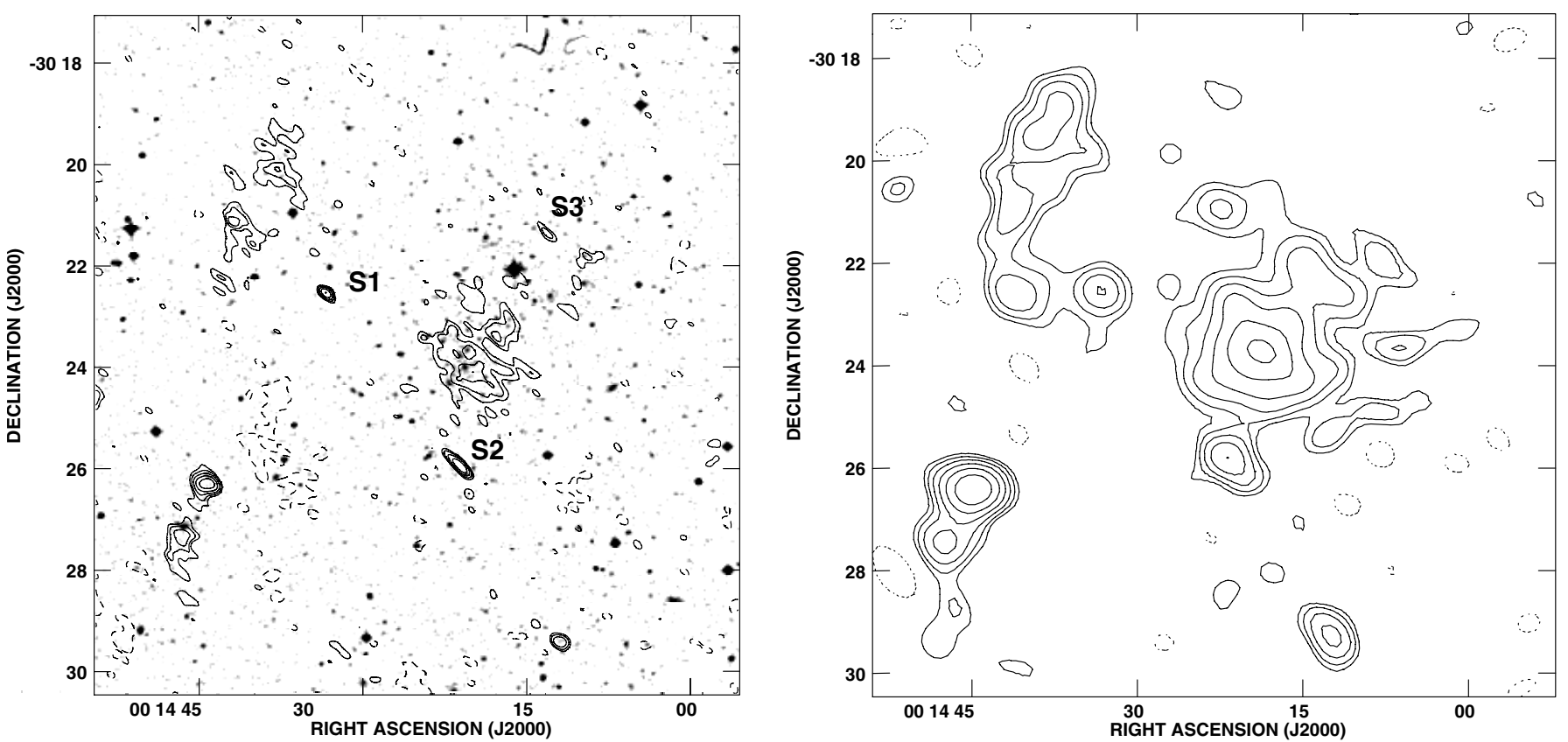

Fig. 1. A2744. Left panel: radio contours of the $325 \mathrm{MHz}$ image obtained with the VLA in BnA configuration are overlaid to the Digitized Sky Surveys optical image of the cluster. The radio image has a resolution of $21^{\prime \prime} \times 11^{\prime \prime}$ with a PA $=51^{\circ}$. The noise level is $0.9 \mathrm{mJy} / \mathrm{beam}$. Contours are scaled by $\sqrt{2}$ and the first two levels are -2 and $2 \mathrm{mJy} /$ beam. Right panel: radio contours of the image at $325 \mathrm{MHz}$ obtained with the VLA in CnB configuration. The resolution is $56^{\prime \prime} \times 44^{\prime \prime}$ with a PA $=58^{\circ}$. The noise level is $2.3 \mathrm{mJy} / \mathrm{beam}$. Contours are scaled by $\sqrt{2}$ and the first two levels are -4.6 and $4.6 \mathrm{mJy} / \mathrm{beam}$.

Table 3. Radio parameters.

\begin{tabular}{ccccc}
\hline \hline Name & type & $\begin{array}{c}S_{325 \mathrm{MHz}} \\
\mathrm{mJy}\end{array}$ & $\alpha$ & $\begin{array}{c}B_{\mathrm{eq}} \\
\mu \mathrm{G}\end{array}$ \\
\hline $\mathrm{A} 2744$ & $\mathrm{H}$ & $218 \pm 10$ & $1.0 \pm 0.1$ & 0.5 \\
& $\mathrm{R}$ & $98 \pm 7$ & $1.1 \pm 0.1$ & 0.6 \\
$\mathrm{~A} 2219$ & $\mathrm{H}$ & $232 \pm 17$ & $0.9 \pm 0.1$ & 0.4 \\
\hline
\end{tabular}

Column 1: cluster name; Col. 2: source type $(\mathrm{R}=$ relic, $\mathrm{H}=$ halo); Col. 3: flux density; the contribution of the point sources have been subtracted; Col. 4: average spectral index between $325 \mathrm{MHz}$ and $1.4 \mathrm{GHz}$; Col. 5: equipartition magnetic field.

of discrete sources. There are only three discrete sources in proximity to the radio halo visible in the $325 \mathrm{MHz}$ image; they are labeled with S1, S2 and S3 in the left panel of Fig. 1. Of these sources, $\mathrm{S} 2$ could be associated with the member galaxy number 52 in Boschin et al. (2006), whereas S1 and S3 do not have any obvious optical identification and are likely background objects. We note here that the strong discrete source detected at $R A=00^{\mathrm{h}} 14^{\mathrm{m}} 4^{\mathrm{s}}$ and $\mathrm{Dec}=-30^{\mathrm{d}} 24^{\mathrm{m}} 41^{\mathrm{s}}$ in the $1.4 \mathrm{GHz}$ image
(Govoni et al. 2001a, see also Fig. 3 bottom left panel) is not detected in the $325 \mathrm{MHz}$ high resolution image of Fig. 1 (left) and is very faint in the lower resolution image of Fig. 1 (right). This is consistent with a source with a spectral index lower than $\alpha<0.5$.

The $\mathrm{CnB}$ configuration image is shown in the right panel of Fig. 1. This image has a resolution of $56^{\prime \prime} \times 44^{\prime \prime}$. The combined $\mathrm{BnA}+\mathrm{CnB}$ array image at $325 \mathrm{MHz}$ (not shown here), produced at intermediate angular resolution $\left(26^{\prime \prime} \times 16^{\prime \prime}\right)$, has a noise level of $1 \mathrm{mJy} / \mathrm{beam}$, and confirms the structures detected from the image of Fig. 1.

The morphology of the diffuse emission is similar to that detected at $1.4 \mathrm{GHz}$ by Govoni et al. (2001a) for both the halo and the relic, although only the brightest regions of the halo can be seen at $325 \mathrm{MHz}$ due to lower sensitivity of these observations with respect to that at $1.4 \mathrm{GHz}$. The radio halo in the center of the cluster has a size of $\sim 6^{\prime}(\sim 1.6 \mathrm{Mpc})$. The relic is located in the North-Eastern region of the cluster and it shows an elongation in North South direction with a size of $\sim 6^{\prime} \times 1^{\prime}(\sim 1.6 \times 0.3 \mathrm{Mpc})$. Table 3 summarizes the main physical parameters obtained from the $325 \mathrm{MHz}$ image both for the halo and the relic. The total flux 


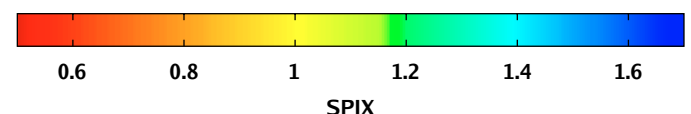

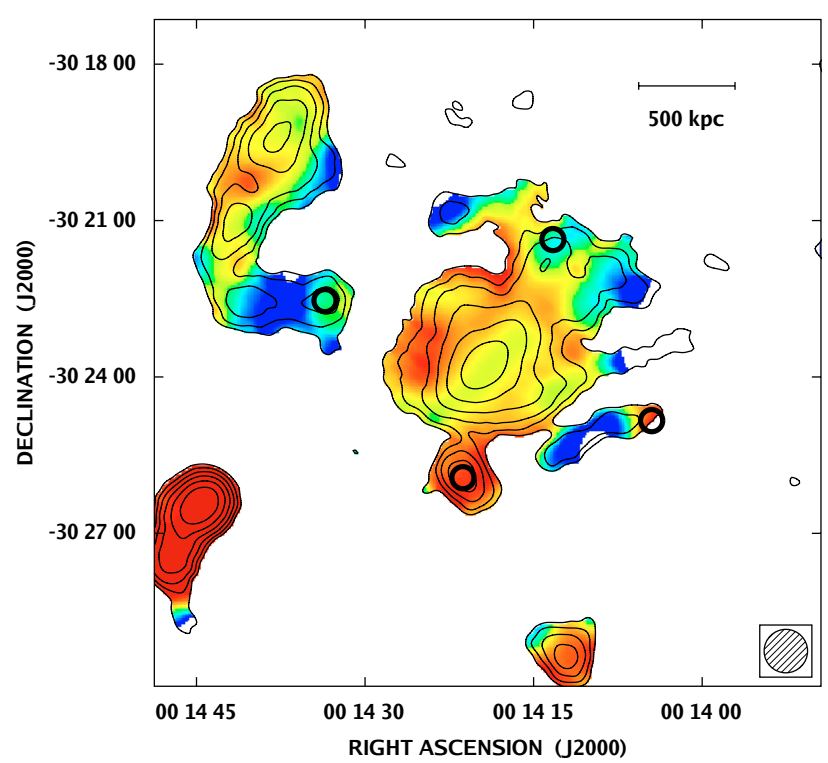

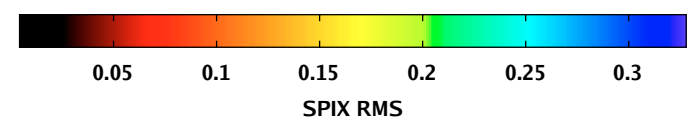

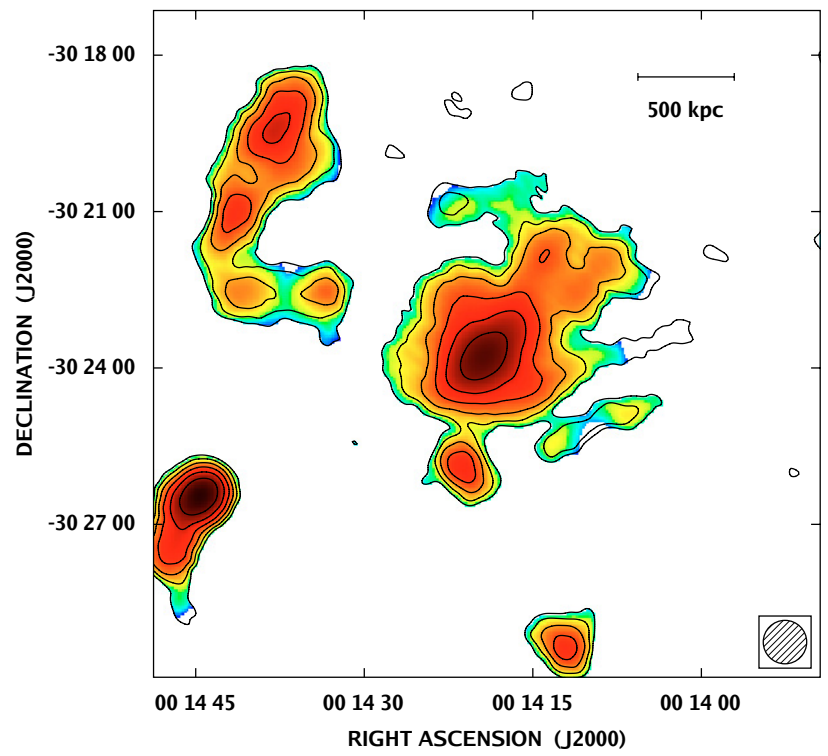

Fig. 2. A2744. Left panel: the color-scale represents the spectral index image of A2744 between $325 \mathrm{MHz}$ and $1.4 \mathrm{GHz}$, with a resolution of $50^{\prime \prime} \times 50^{\prime \prime}$. Pixels whose brightness was below $3 \sigma$ at $325 \mathrm{MHz}$ or $1.4 \mathrm{GHz}$ have been blanked. The cut is driven by the $325 \mathrm{MHz}$ image in most of the points. Contour levels are the radio image at $325 \mathrm{MHz}$. Contours start at $3.9 \mathrm{mJy} /$ beam $(3 \sigma)$ and scale by $\sqrt{2}$. The circles indicate the positions of discrete sources, the spectral index in these points is not representative of the radio halo emission. Right panel: the color-scale represents the image of the spectral index uncertainty.

of the halo, excluding discrete sources, is $218 \pm 10 \mathrm{mJy}$. Using the total flux at $325 \mathrm{MHz}$ and $1.4 \mathrm{GHz}$, measured in the same area, we calculated an average total spectral index of the halo of $\alpha \sim 1 \pm 0.1$. The total flux of the relic is $98 \pm 7 \mathrm{mJy}$ and its average spectral index is $\alpha \sim 1.1 \pm 0.1$.

\subsection{Spectral index analysis of $A 2744$}

For the purposes of the spectral analysis we combined the BnA and $\mathrm{CnB}$ arrays at $325 \mathrm{MHz}$ and we compared this image with the $\mathrm{C}+\mathrm{D}$ observation at $1.4 \mathrm{GHz}$ by Govoni et al. (2001). Images at both frequencies have been restored with the same beam of $50^{\prime \prime} \times 50^{\prime \prime}$. The final noise levels are 1.3 and $0.1 \mathrm{mJy} /$ beam for the 325 and $1.4 \mathrm{GHz}$ images, respectively.

The spectral index image of A2744, calculated between $325 \mathrm{MHz}$ and $1.4 \mathrm{GHz}$, is shown in left panel of Fig. 2. In the figure we indicate by circles the discrete sources, whose spectrum is not related to the radio halo. Right panel of Fig. 2 shows the spectral index uncertainty, $\sigma_{\alpha}$. The spectral index image has been obtained by considering only those pixels where the brightness was above $3 \sigma$ level at both frequencies. Because of its higher noise, the cut is driven by the $325 \mathrm{MHz}$ image in most of the points. The very different sensitivities of the $325 \mathrm{MHz}$ and $1.4 \mathrm{GHz}$ images introduce a bias in the spectral index image. Indeed, the outermost source regions, whose brightness at $1.4 \mathrm{GHz}$ is at the lowest levels, can only be detected at $325 \mathrm{MHz}$ if their spectrum is steeper than $\sim 1.8$ (see discussion below).

The spectral index image is patchy. The central region has a spectral index of $\alpha \simeq 1.05 \pm 0.04$. A prominent region of flatter spectrum is present toward the East, with values of $\alpha \simeq 0.7 \pm 0.1$. Another flat spectrum region is located North-West, before the asymmetric halo extension. Beyond this region, the spectrum is steep with values of the spectral index $\alpha \simeq 1.5 \pm 0.2$. By comparison of the spectral index image with the error image (Fig. 2 left and right panel, respectively) it is evident that most spectral index features are statistically significant.

In the relic, the spectral index trend shows a gradual steepening from values of $\alpha \sim 0.9 \pm 0.1$ to $\alpha \sim 1.6 \pm 0.2$ from the outer to the inner rim. There are not drastic gradients along the main axis, as for the relics in A2256 (Clarke \& Enßlin 2006) and A3667 (Rottgering et al. 1997). Hoeft \& Brueggen (2007) have shown that an overall slope slightly steeper than 1 is inevitable for a merger induced relic.

We are interested to investigate if there is a systematic variation of the radio halo spectral index with radius as found in the radio halos of Coma (Giovannini et al. 1993), A665 and A2163 (Feretti el al. 2004). The spectral index presented in Fig. 2 is fully sampled to within a distance of about one core radius from the cluster center $\left(r_{\mathrm{c}} \simeq 115^{\prime \prime} \equiv 520 \mathrm{kpc}\right.$; Govoni et al. 2001). Since the $325 \mathrm{MHz}$ image has a noise level which is an order of magnitude higher than the $1.4 \mathrm{GHz}$ image, only the brightest regions of the radio halo at $325 \mathrm{MHz}$ can be detected beyond this distance. As stated above, the regions of the lowest $1.4 \mathrm{GHz}$ brightness are only detected if their spectrum is steeper than $\alpha>1.8$, otherwise their $325 \mathrm{MHz}$ emission is too faint to be detected at the sensitivity limit of the $325 \mathrm{MHz}$ image. As a consequence, a radial steepening of the halo spectrum would be undetectable in the spectral index image, unless it is very strong, to values of $\alpha>1.8$ in the peripheral regions of the halo. Thus, to improve the statistics and take into account the limits on the $325 \mathrm{MHz}$ emission, we derived the radial trend of the spectral index from the azimuthally averaged brightness profiles at $1.4 \mathrm{GHz}$ and $325 \mathrm{MHz}$ without imposing any cut on the radio images. In order to improve the statistics, we averaged the brightness in ten concentric annuli of $\sim 25^{\prime \prime}$ in width centered on the radio peak, as shown in top-left panel of Fig. 3. The resulting azimuthally 

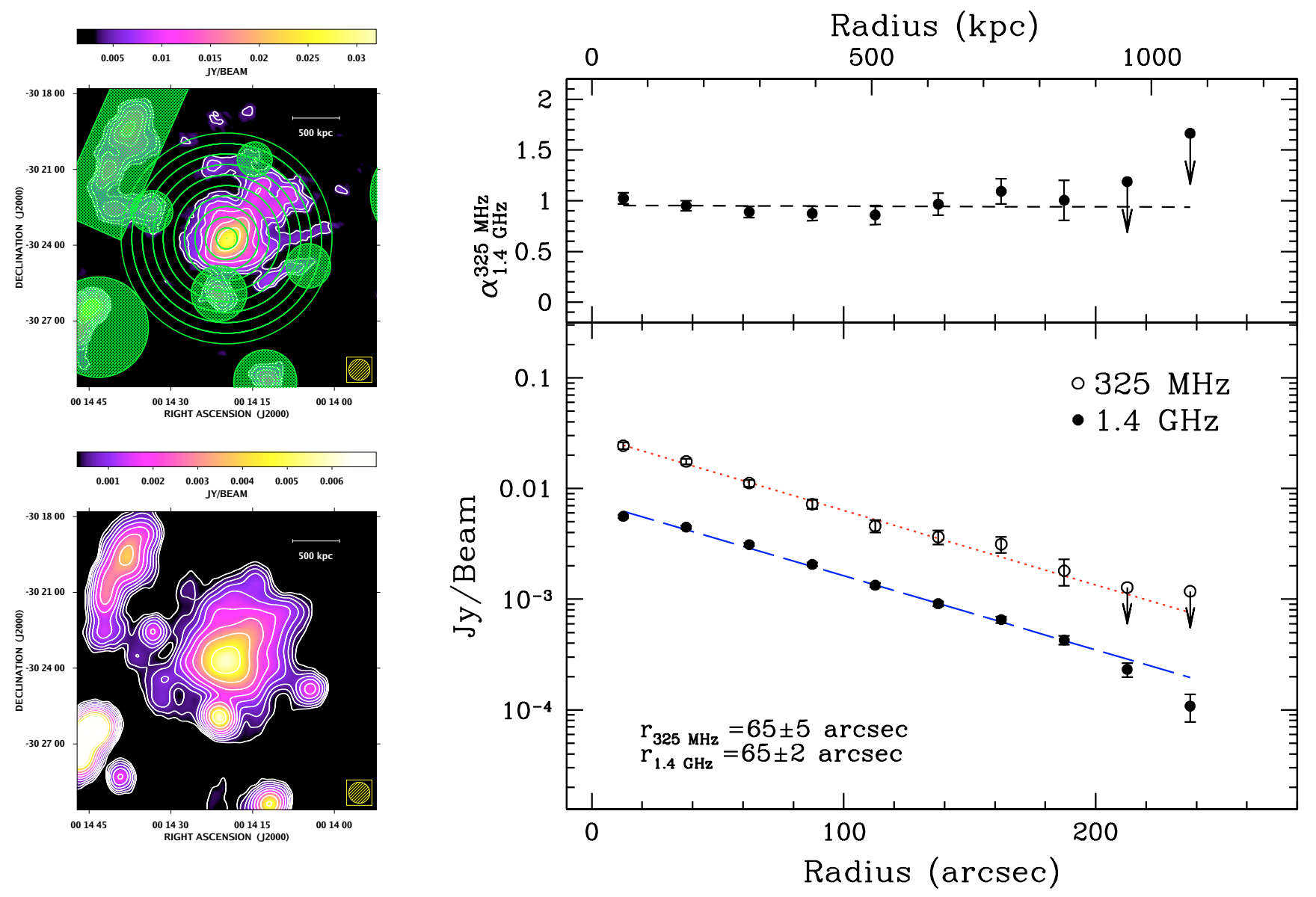

Fig. 3. A2744. Left: colors represent the images at $325 \mathrm{MHz}$ (top panel) and $1.4 \mathrm{GHz}$ (bottom panel). Contours represent the $325 \mathrm{MHz}$ and $1.4 \mathrm{GHz}$ images. Contours are spaced by $\sqrt{2}$ and start at 3.9 and $0.3 \mathrm{mJy} /$ beam at $325 \mathrm{MHz}$ and $1.4 \mathrm{GHz}$, respectively. The cross-hatched regions have been excluded from the statistics. Right: bottom panel shows the azimuthally averaged brightness profiles at $325 \mathrm{MHz}$ (open circles) and at $1.4 \mathrm{GHz}$ (solid circles). Arrows represent $3 \sigma$ upper-limits. The lines represent the fit of an exponential profile to the data (see the text). In the top panel we plot the spectral index radial profile obtained from the brightness profiles.

averaged brightness and spectral index profiles are shown in the right panel of Fig. 3. The errors associated with each point represent the $1 \sigma$ error on the average value while arrows represent $3 \sigma$ upper-limits.

Brightness values below a level of $3 \sigma$ have been considered upper-limits. We fitted the brightness profiles with an exponential law of the form

$I(r)=I_{0} \mathrm{e}^{-r / r_{v}}$

and we derived $r_{v}$, the e-folding radius of the brightness profile at both frequencies. It results $r_{325 \mathrm{MHz}}=65^{\prime \prime} \pm 5^{\prime \prime}$ and $r_{1.4 \mathrm{GHz}}=65^{\prime \prime} \pm 2^{\prime \prime}$. The e-folding radius at $325 \mathrm{MHz}$ is equal, to within the errors, to that at $1.4 \mathrm{GHz}$, this implies that the azimuthally averaged spectral index is constant with the increasing distance from the cluster center as shown in the top plot of right panel of Fig. 3. Here, the points represent the spectral index obtained from the azimuthally average brightness profiles and the dashed line represents the trend expected from the fit of the exponential law. The azimuthally averaged spectral index in A2744 is constant to a value close to $\alpha \simeq 1$ up to a distance of $1 \mathrm{Mpc}$ from the cluster center. This result implies that, although there are significant variations of the spectral index from pointto-point, on average the radio spectrum of the radio halo does not change with radius.

In Fig. 4 we show the analysis of the azimuthally average brightness profiles for four cluster quadrants. We note that within the core radius $\left(r_{\mathrm{c}}=115^{\prime \prime}\right) \alpha \simeq 1$ for all the four quadrants. However, at larger distances from the cluster center the four profiles differ. In agreement with the spectral index image, the steepest spectra are detected in the NW quadrant. Here, the spectral index reaches values of $\alpha \simeq 1.6 \pm 0.2$, after some flattening at about $80-120^{\prime \prime}$. The flattest spectra are found in the SE sector, where the values of the outermost regions are constrained by upper limits.

\subsection{Equipartition magnetic field}

Under the assumption that a radio source is in a minimum energy condition, it is possible to derive a zero-order estimate of the magnetic field strength averaged over the entire source volume. In the classical equipartition assumption, considering a simplified shape of the spectrum of the emitting electrons in the form:

$N(\varepsilon) \mathrm{d} \varepsilon=N_{0} \varepsilon^{-\delta} \mathrm{d} \varepsilon$

the condition of minimum energy is obtained when the relativistic particle energy density $\varepsilon_{\mathrm{CRe}}=\int \varepsilon N(\varepsilon) \mathrm{d} \varepsilon$ is approximately equal to the magnetic field energy density $\varepsilon_{B}=B^{2} /(8 \pi)$. In this assumption the magnetic field can be determined from the radio synchrotron luminosity and the source volume. The volume averaged magnetic field was evaluated within an ellipsoid assuming a magnetic field entirely filling the radio source, equal energy 

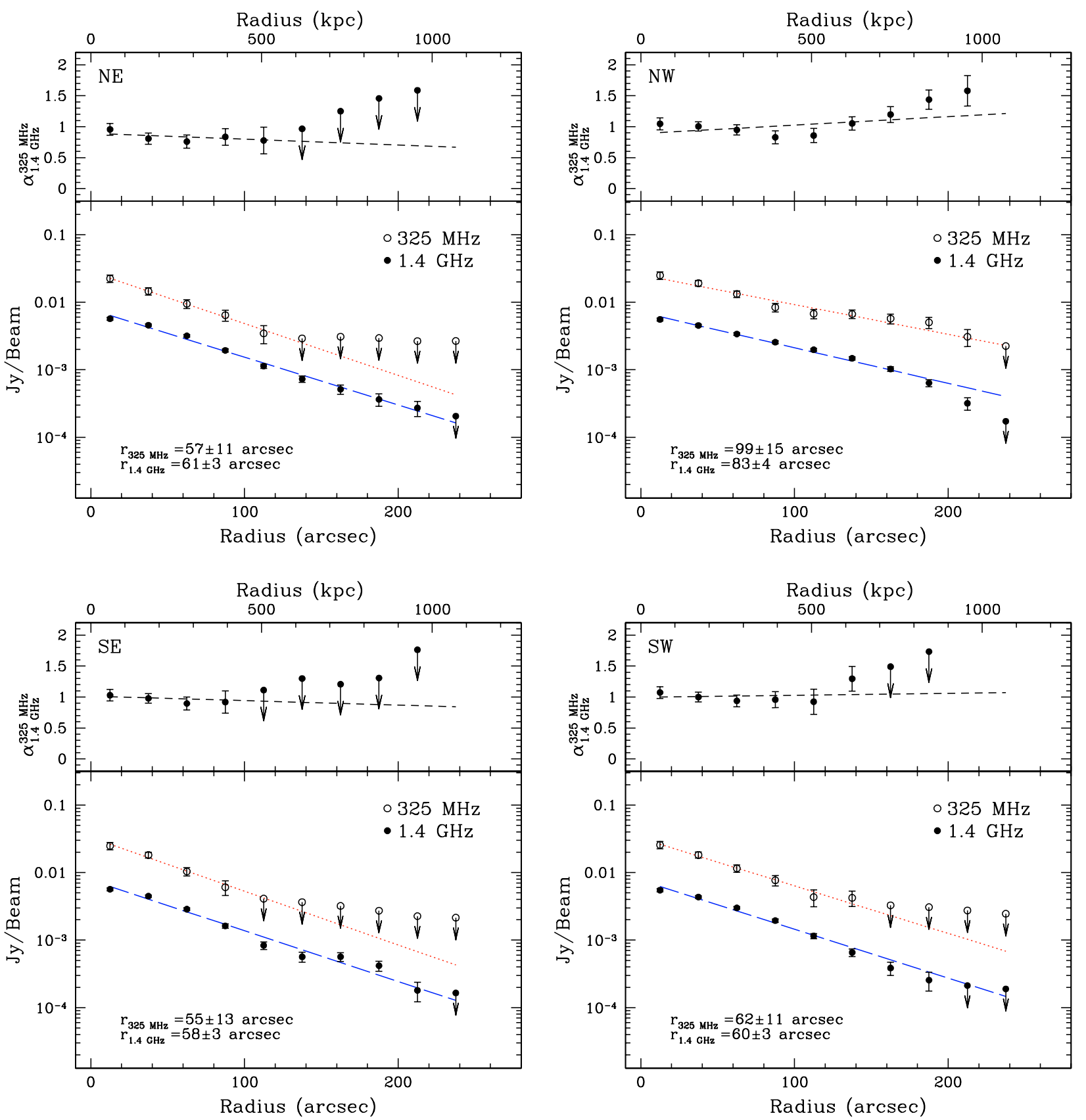

Fig. 4. A2744. Azimuthally averaged brightness profiles for four sectors. Arrows represent $3 \sigma$ upper-limits. The lines represent the fit of an exponential profile to the data (see the text).

in relativistic protons and electrons, and a range of frequencies in which the synchrotron luminosity is calculated from a low frequency cutoff of $10 \mathrm{MHz}$ to a high frequency cutoff of $10 \mathrm{GHz}$. For the spectral index of the electron energy spectrum of the halo we adopted the averaged emission spectral index $\alpha \simeq 1.0$ which yields $\delta=3.0$. For the relic we use $\alpha \simeq 1.1$ which yields $\delta=3.2$. We estimated an equipartition magnetic field $B_{\text {eq }} \sim 0.5 \mu \mathrm{G}$ in the halo and $B_{\text {eq }} \sim 0.6 \mu \mathrm{G}$ in the relic.

Assuming a low-frequency cut-off of $10 \mathrm{MHz}$ in the luminosity calculation is equivalent to assuming a low-energy cutoff of $\gamma_{\min } \sim 2000$ in the particle energy spectrum. If alternatively we adopt a low-energy cut-off of $\gamma_{\min }=100$ in the particle energy distribution rather than a low-frequency cut-off in the emitted synchrotron spectrum (e.g. Brunetti et al. 1997;
Beck \& Krause 2005), we obtain $B_{\mathrm{eq}}^{\prime} \sim 1.0 \mu \mathrm{G}$ in the halo and $B_{\mathrm{eq}}^{\prime} \sim 1.3 \mu \mathrm{G}$ in the relic.

The radio synchrotron emissivity is given by:

$j_{v} \propto N_{0} B^{(\delta+1) / 2} v^{-(\delta-1) / 2}$.

Under equipartition conditions, if $\gamma_{\min }$ is assumed constant with cluster radius, it is $N_{0} \propto B^{2}$ and therefore:

$j_{v} \propto B^{(\delta+5) / 2}$.

According to this relation, assuming $\delta=3$, a spherical symmetry and using the de-projected brightness profile at $325 \mathrm{MHz}$, we obtain the equipartition magnetic field radial trend shown in Fig. 5. In each annulus, the de-projected profile has been obtained by subtracting the brightness contribution of the external 


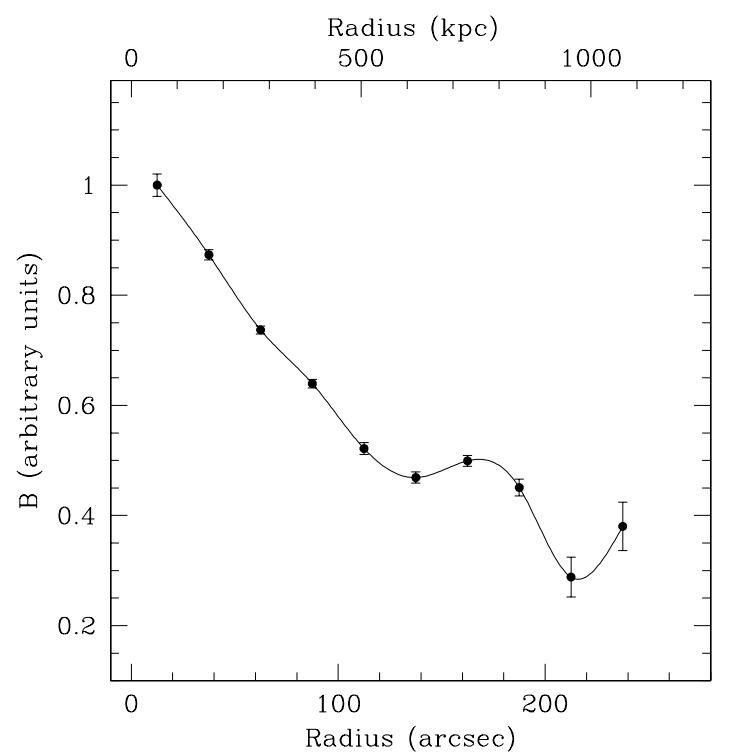

Fig. 5. Equipartition magnetic field radial profile obtained for the halo in $\mathrm{A} 2744$ for $\delta=3$. The profile has been normalized to its value at the center.

shells. The magnetic field strength decreases by a factor of two from the cluster center to the halo periphery.

\subsection{Spectral index versus optical and $X$-ray bands.}

On the basis of optical and X-ray data from the Chandra satellite, the following merger scenario has been proposed for A2744 (Girardi \& Mezzetti 2001; Kempner \& David 2004; Boschin et al. 2006): a main merger has been observed, in the proximity of the cluster center, principally along the line of sight, in which two sub-clusters with mass ratio $3: 1$ and a non-zero impact parameter are interacting. A peripheral merger, with evidence of bow shock in the X-ray image, has been seen in the North-Western region. It involves a less massive sub-cluster and the central region in which the main merger is occurring.

Recent calculations (Cassano \& Brunetti 2005) show that a fraction $\sim 10 \%$ of the thermal-cluster energy may be channeled in the form of turbulence in case of main (i.e., mass ratio $\leq 5: 1$ ) cluster mergers and this may power up giant radio halos such as observed in the case of A2744. Although the minor mergers cannot directly generate giant radio halos, they may still power the particle acceleration process especially if they happen in clusters that are already dynamically disturbed by previous mergers.

On the basis of the above merger scenario, we investigated if there is any connection between the merger activity observed in the optical and X-ray bands and the spectral index distribution in the radio halo.

The recent optical analysis by Boschin et al. (2006) based on photometric and spectroscopic data of cluster galaxies shows that A2744 is a very complex cluster from the optical point of view. They pointed out a displacement between the peak of galaxy distribution (see also the weak lensing analysis by Smail et al. 1997), and the peak of X-ray emission, as expected in the case of cluster mergers from numerical simulations (e.g., Roettiger et al. 1997). The peak of radio emission is displaced with respect to both optical and X-ray centers, reinforcing the idea that this cluster is dynamically very disturbed.

The two-dimensional distribution of likely members galaxies from Boschin et al. (2006) is shown in contours in the left panel of Fig. 6. Galaxies are concentrated in two regions: a main clump, located at the center of the cluster, and a secondary clump is placed at about $2.5^{\prime}$ North with respect to the cluster center. Spectroscopically these galaxies are characterized by two velocity groups separated by a line-of-sight velocity of $\Delta V \sim$ $4000 \mathrm{~km} \mathrm{~s}^{-1}$. The main one, the low-velocity group, has a velocity dispersion of $\sigma_{V} \simeq 1200-1300 \mathrm{~km} \mathrm{~s}^{-1}$. These galaxies are indicated with open boxes in left panel of Fig. 6 and are distributed over the whole cluster. The secondary one, the high-velocity group, has a velocity dispersion of $\sigma_{V} \simeq 500-800 \mathrm{~km} \mathrm{~s}^{-1}$. The galaxies of the high-velocity group are indicated with filled circles in left panel of Fig. 6 and are mainly concentrated in the South-West of the cluster. The high-velocity group is likely merging with the main system and being responsible for the strongly disturbed central region. From the comparison with the spectral index image, there is no evident association between optical and spectral features. However, from the radial profiles presented in Fig. 4, it is derived that the southern flat spectrum part of the radio halo coincides in projection with the high-velocity group of galaxies.

A spatial comparison between the spectral index image of A2744 and the Chandra X-ray brightness image (courtesy of Kempner, see Kempner \& David 2004) is shown in the right panel of Fig. 6. There is no evident correlation between the radio spectral index and the X-ray brightness features at the cluster center. We note, however, that the region of the NW group has a steep spectrum, while the stripe between the cluster center and the NW group has a spectrum significantly flatter than the cluster center and the group itself. This is likely the region affected by the collision between the main cluster and the group.

We further compared the spectral index of the radio halo and the gas temperature image (kindly supplied by Kempner) of A2744. We averaged the spectral index and the temperature using a grid of rectangular boxes $63^{\prime \prime} \times 63^{\prime \prime}$ in size (see left panel of Fig. 7), which is the extraction region of the temperature image (Kempner \& David 2004). The resulting scatter plot is shown in the right panel of Fig. 7 where each point represents the average value in each cell of the grid. We found that the region with the highest gas temperature $(T \simeq 10 \mathrm{keV})$ coincides with the flat spectrum clump in the radio halo $(\alpha \simeq 0.7)$ and that, in general, steep spectrum regions tend to be associated with lower temperature. This is the first time that such a correlation is detected in a radio halo.

\section{Properties of the cluster A2219}

A2219 hosts a giant radio halo detected in the NVSS by Giovannini et al. (1999) and confirmed in a deeper observation at $1.4 \mathrm{GHz}$ by Bacchi et al. (2003). In the cluster center there are three strong radio sources identified as cluster galaxies by Owen et al. (1992). Using Chandra archive X-ray data and optical spectra obtained with the $T N G$, Boschin et al. (2004) confirmed that the cluster is not dynamically relaxed. Indeed this cluster shows a SouthEast-NorthWest elongation, that is supported by the spatial distribution of the color-selected likely cluster members, the shape of the $\mathrm{cD}$ galaxy, the X-ray contours levels, the gradient in the velocity dispersion and the X-ray temperature. From multi-wavelength analysis a very complex scenario results for the dynamic of the merger. Boschin et al. (2004) suggested that the $\mathrm{cD}$ galaxy in the center is undergoing the consecutive merger of many clumps aligned in a filament for which the projection along the line of view is obliquely oriented. They found a possible confirmation of this scenario in the elongated shape in 

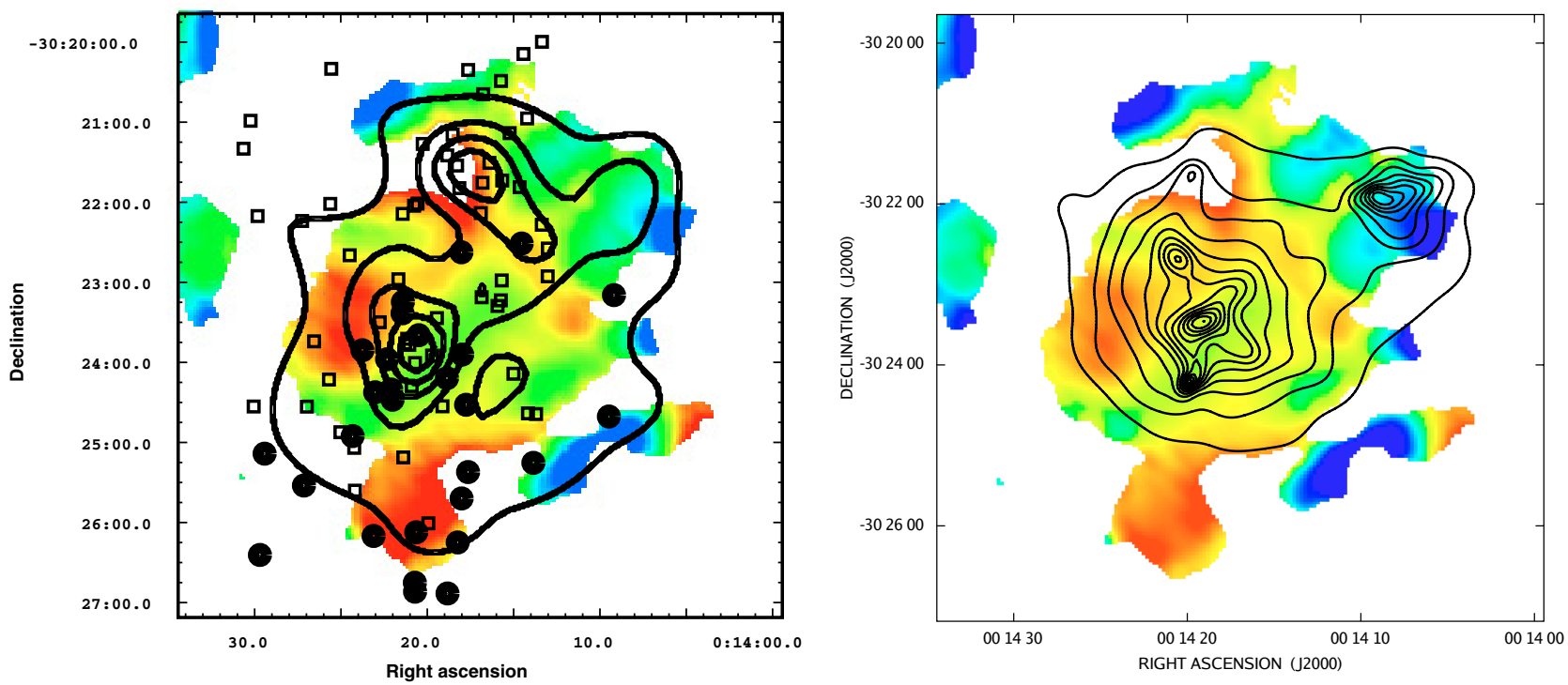

Fig. 6. A2744: The spectral index images are shown in colors. Left panel: contours are the spatial 2D distribution of likely members galaxies taken Boschin et al. (2006). Solid circles and open boxes represent the high-velocity and low-velocity galaxies, respectively. Right panel: contours are the Chandra X-ray image, by Kempner \& David (2004).
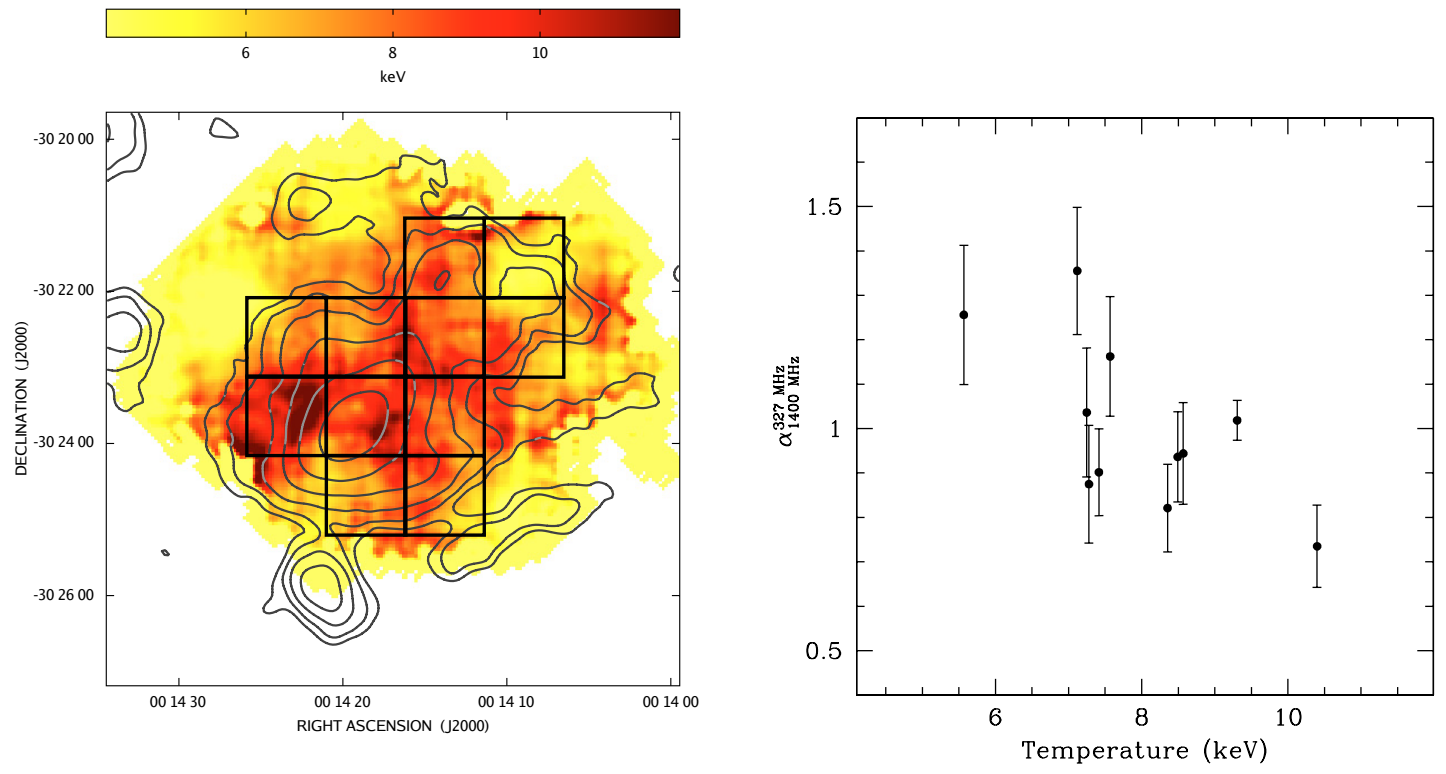

Fig. 7. Left panel: the grid used to calculate the spectral index-temperature scatter plot is overlayed on the Chandra temperature image (gray scale) by Kempner \& David (2004). The contours represent the $325 \mathrm{MHz}$ image. Right panel: scatter plot of the radio halo spectral index between $325 \mathrm{MHz}$ and $1.4 \mathrm{GHz}$ versus gas temperature.

the South East-North West direction observed in the radio contours map at $1.4 \mathrm{GHz}$ by Bacchi et al. (2003).

\subsection{Low frequency image of $A 2219$}

The A2219 diffuse radio emission at $325 \mathrm{MHz}$ obtained with the VLA in C configuration is shown in the image of Fig. 8 (left panel), which has a sensitivity level of $1.7 \mathrm{mJy} /$ beam and a resolution of $56^{\prime \prime} \times 53^{\prime \prime}$. The obtained sensitivity allows good detection of the central region of about $\sim 4.5^{\prime} \quad(\sim 950 \mathrm{kpc})$ of the halo, in which the morphology is similar to that shown by
Bacchi et al. (2003) with a North West-South East elongation. The sensitivity of our observation is not high enough to detect more extended regions as seen in low resolution images at 1.4 GHz in Bacchi et al. (2003).

In the right panel of Fig. 8 we show a zoom of the central region of A2219, obtained with the VLA in B configuration, overlaid on the $R$-band optical image (Boschin et al. 2006). The sensitivity level is $1.7 \mathrm{mJy} /$ beam, the resolution is $18^{\prime \prime} \times 16^{\prime \prime}$. The radio emission at the cluster center is the blend of three cluster radio galaxies. 


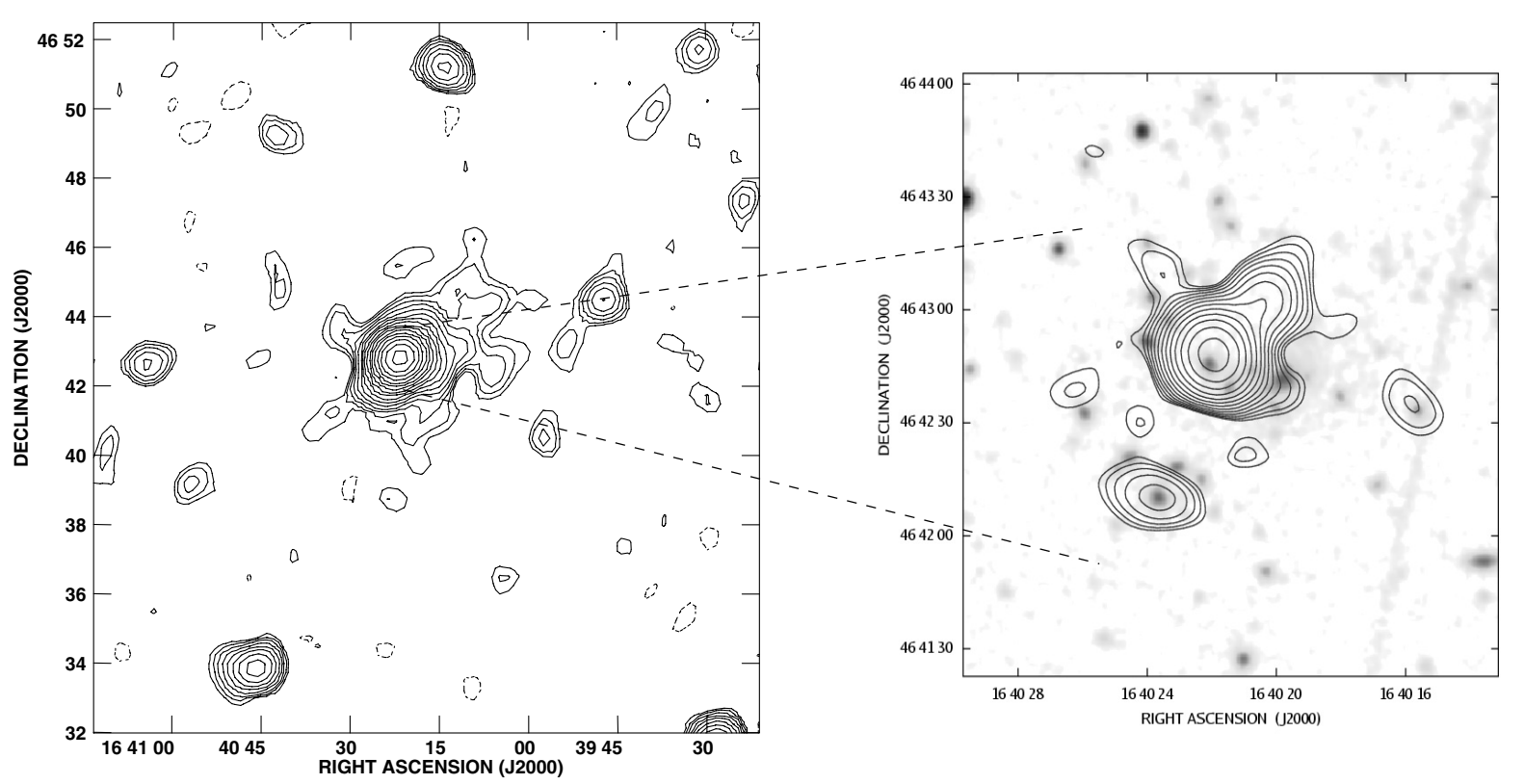

Fig. 8. A2219. Left panel: radio contours of the image at $325 \mathrm{MHz}$ obtained with the VLA in C configuration. The resolution is $56^{\prime \prime} \times 53^{\prime \prime}$ with $\mathrm{PA}=-85^{\circ}$. The noise level is $1.7 \mathrm{mJy} /$ beam, contours are scaled by $\sqrt{2}$ where the first two levels are -3.4 and $3.4 \mathrm{mJy} / \mathrm{beam}$. Right panel: radio contours of the image at $325 \mathrm{MHz}$ obtained with the VLA in B array overlaid onto the optical image (from Boschin et al. 2006). The resolution of the the radio image is $18^{\prime \prime} \times 16^{\prime \prime}$ with $\mathrm{PA}=41^{\circ}$, the noise level is $1.7 \mathrm{mJy} /$ beam, contours start at $7.5 \mathrm{mJy} / \mathrm{beam}(4.5 \sigma$ level $)$ and are scaled by $\sqrt{2}$.

The total flux, after the subtraction of discrete sources is $232 \pm 17 \mathrm{mJy}$ and the average spectral index of the halo, calculated between $325 \mathrm{MHz}$ and $1.4 \mathrm{GHz}$, is $\alpha \sim 0.9 \pm 0.1$.

The equipartition magnetic field, calculated within a sphere of $330^{\prime \prime}$ of radius following the classical approach, is $B_{\text {eq }} \sim$ $0.4 \mu \mathrm{G}$ (for $\delta=2.8$ ) and $B_{\text {eq }}^{\prime} \sim 0.7 \mu \mathrm{G}$ (assuming $\gamma_{\text {min }}=100$ ) with the approach given in Brunetti et al. (1997).

The main physical parameters of the radio halo in A2219 are summarized in Table 3.

\subsection{Spectral index analysis of $A 2219$}

The spectral index image of A2219 with a resolution of $56^{\prime \prime} \times 56^{\prime \prime}$ is shown in left panel of Fig. 9. The spectral index image, calculated between $325 \mathrm{MHz}$ and $1.4 \mathrm{GHz}$, have been made using images with the same restored beam, with the same pixel size and considering only pixels with brightness values above $3 \sigma$ at both frequencies. The right panel of Fig. 9 shows the $1-\sigma$ spectral index uncertainty.

The spectral index is $\alpha \sim 0.8 \pm 0.05$ in the inner $300 \mathrm{kpc}$, where three blended compact sources are present. At larger distances, the spectral index can be considered representative of the halo emission and ranges between $\alpha=1.0$ and $\alpha=1.5$ with a typical uncertainty of 0.2 .

In Fig. 10 (right), the brightness and the spectral profiles for the halo in A2219 are presented. The notation is the same as in Fig. 3. As mentioned before, since in A2219 the radio emission in the center of the halo is dominated by the blend of three point sources, we focused only on the brightness and spectrum at distances more than $500 \mathrm{kpc}$ from the cluster center. The azimuthally averaged spectral index in the radio halo of A2219 is constant within the error, with an average value of $\alpha \simeq 1$.

Due to the limited information on this radio halo, no attempt was made to derive constraints on the equipartition magnetic field profile for this cluster.
As for A2744, we compare results from radio observations to those from the recent optical analysis based on photometric and spectroscopic data for member galaxies (Boschin et al. 2004). The radio center coincides with the center of the galaxy distribution, i.e. the position of the $\mathrm{cD}$ galaxy. The western extension in the radio emission has an interesting possible correspondence in the western extension of the 2D spatial distribution of bright galaxies (see Fig. 9 middle panel of Boschin et al. 2004) and in the East-West direction of the global velocity gradient as recovered from the spectroscopic data.

\section{Discussion}

One of the main difficulties in explaining radio halos arises from the combination of their Mpc size and the short radiative lifetime of the relativistic electrons (about $10^{8} \mathrm{yr}$ ): the diffusion time necessary for these electrons to cover such distances is much larger than their radiative lifetime. Thus, it is required that either the electrons are re-accelerated (primary models) or continuously injected over the entire cluster volume by hadronic collisions (secondary models). Detailed studies of the spectral index in radio halos can provide important inputs to the above models.

For instance, secondary models assume that the relativistic electrons are continuously injected by cosmic ray protons colliding with thermal protons. The cosmic ray protons are accelerated, with a power law energy spectrum, by cluster merger shocks and/or by galactic winds and then they are accumulated over cosmological epochs during the cluster formation (e.g. Berezinsky et al. 1997; Pfrommer et al. 2006, and references therein). The energy spectrum of the relativistic electrons produced by the diffusion of the cosmic ray protons through the ICM is expected to be a smooth power law. In these models, variations in the halo's magnetic field strength do not produce variation in the radio spectral index. Thus, the constancy of the azimuthally average spectral index profile observed in A2744 and A2219 is in agreement with the expectation of secondary 

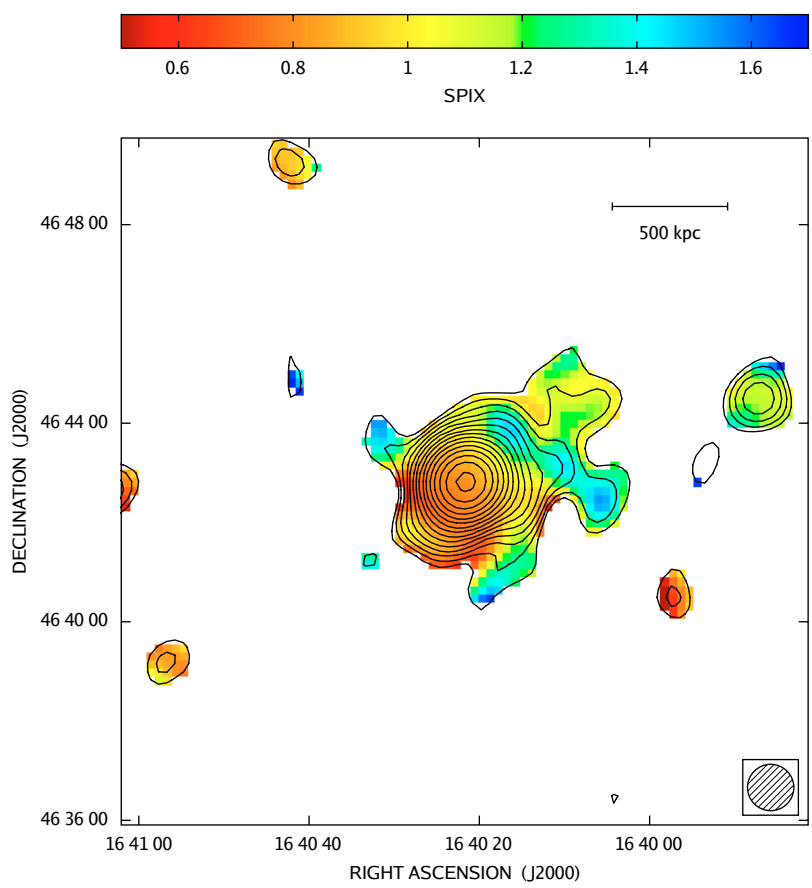
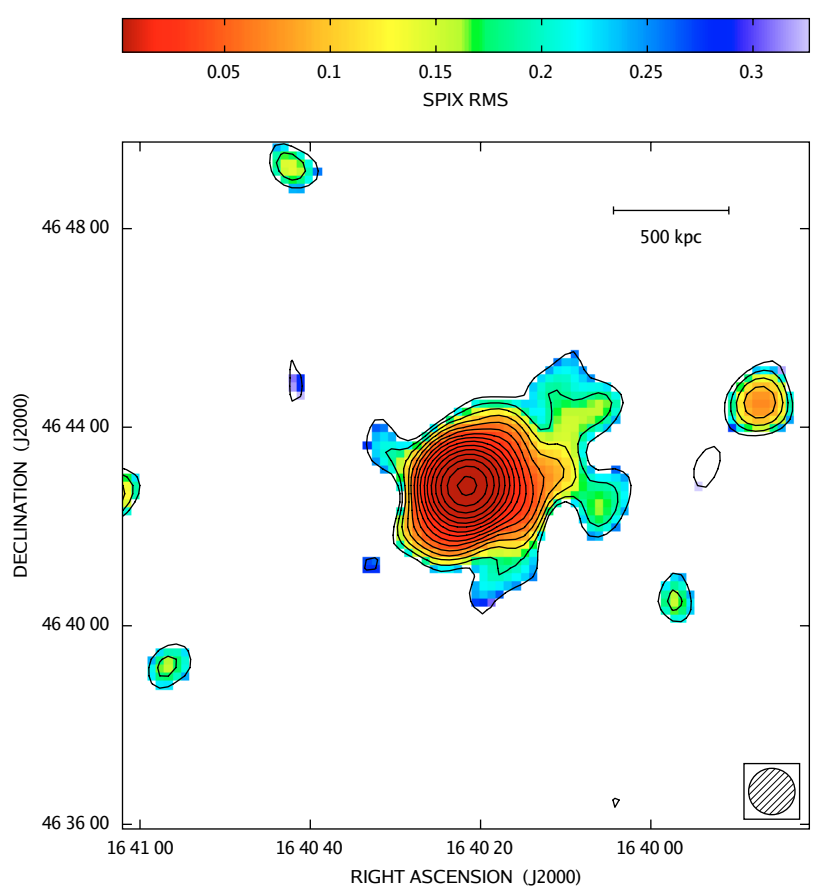

Fig. 9. A2219. Left panel: the color-scale represents the spectral index image of A2219 between $325 \mathrm{MHz}$ and $1.4 \mathrm{GHz}$, with a resolution of $56^{\prime \prime} \times 56^{\prime \prime}$. Pixels whose brightness was below $3 \sigma$ at $325 \mathrm{MHz}$ or $1.4 \mathrm{GHz}$ have been blanked. The cut is driven by the $325 \mathrm{MHz}$ image in most of the points. Contour levels are the radio image at $325 \mathrm{MHz}$. Contours start at $5.1 \mathrm{mJy} / \mathrm{beam}(3 \sigma)$ and scale by $\sqrt{2}$. Right panel: the color-scale represents the image of the spectral index uncertainty.

models. However, the patchy structure of the spectral index image in A2744 shows significant variations of the radio halo spectrum over distances as low as $\sim 200 \mathrm{kpc}$. These variations cannot be explained by a featureless power law energy spectrum for the synchrotron electrons and indicate that more complex processes are at work in the ICM.

Primary models assume that the relativistic electrons are reaccelerated over the entire cluster. The particle re-acceleration can be powered by the energy dissipated during cluster mergers. The anti-correlation between spectral index and gas temperature shown in Fig. 7 supports the idea that a fraction of the gravitational energy, which is dissipated during major and minor mergers in heating the thermal plasma, is converted into re-acceleration of relativistic particles and amplification of the magnetic field. In principle the re-acceleration of these particles may occur either via shock acceleration or via turbulent re-acceleration; both scenarios being qualitatively consistent. However, the lack of a clear morphological connection between the presence of shocks in the X-ray image and of synchrotron emitting regions with flatter spectrum would apparently disfavor the shock hypothesis.

Complex spectral energy distributions for the synchrotron electrons are expected in primary models (Brunetti et al. 2001). Therefore, magnetic field variations within the radio halo can give rise to spectral index patterns such as those observed in the case of A2744. Primary models assume that electrons are re-accelerated up to a maximum energy $\left(\gamma_{\max }\right)$ which marks the balance between acceleration efficiency and energy losses. Above $\gamma_{\max }$ an exponential cut-off in the electron energy spectrum develops. The synchrotron emission extends up to a peak frequency $v_{\text {peak }} \propto \gamma_{\max }^{2} B$, therefore the lower are $\gamma_{\max }$ and/or $B$, the steeper is the synchrotron spectrum measured between two fixed frequencies. In the re-acceleration scenario via the classical Fermi II mechanism, the systematic energy gain of particles becomes significant after one acceleration time-scale, $\tau_{\text {acc }}$. If $\tau_{\text {acc }}$ does not depend on the particle energy, the maximum energy of the accelerated particles essentially depends on the ratio between the acceleration efficiency $\left(\propto \tau_{\text {acc }}^{-1}\right)$ and the particle energy losses:

$\gamma_{\max } \propto \frac{1}{\left(B^{2}+B_{\mathrm{cmb}}^{2}\right) \tau_{\mathrm{acc}}}$

where $B_{\mathrm{cmb}}=3.2 \cdot(1+z)^{2} \mu \mathrm{G}$ is the inverse Compton equivalent field ${ }^{3}$.

In this case the peak frequency scales as:

$v_{\text {peak }} \propto \frac{B}{\left(B^{2}+B_{\mathrm{cmb}}^{2}\right)^{2} \tau_{\mathrm{acc}}^{2}}$

In the simplified hypothesis the acceleration efficiency is constant throughout the cluster and the magnetic field is smaller than the inverse Compton equivalent field, $v_{\text {peak }} \propto B$. Thus, we can infer the variations in the magnetic field strength along those directions in which the observed spectral index shows the maximum changes, i.e. in the NW sector of A2744. The shape of the energy spectrum of the synchrotron electrons depends on the adopted value for $\tau_{\text {acc }}$ and on the time for which the electrons are accelerated (e.g., Ohno et al. 2002; Brunetti et al. 2004; Brunetti \& Blasi 2005; Cassano \& Brunetti 2005; Cho \& Lazarian 2006). Here we calculate the energy distribution of the re-accelerated electrons by assuming $\tau_{\text {acc }}=10^{8} \mathrm{yr}$ (which is appropriate for the emitting particles in radio halos) and by assuming that electrons are re-accelerated for a time-scale $\tau_{H} \sim 3 \tau_{\text {acc }}$ which corresponds to $0.3 \mathrm{Gyr}$ (in line with the age of radio halos, e.g. Hwang 2004). In Fig. 11 we report the radial behavior of the magnetic field strength obtained by fitting the spectral index profile in the North West quadrant of A2744. In the approximation of constant

${ }^{3}$ At the redshift of $\mathrm{A} 2744 B_{\mathrm{cmb}}=5.5 \mu \mathrm{G}$. 

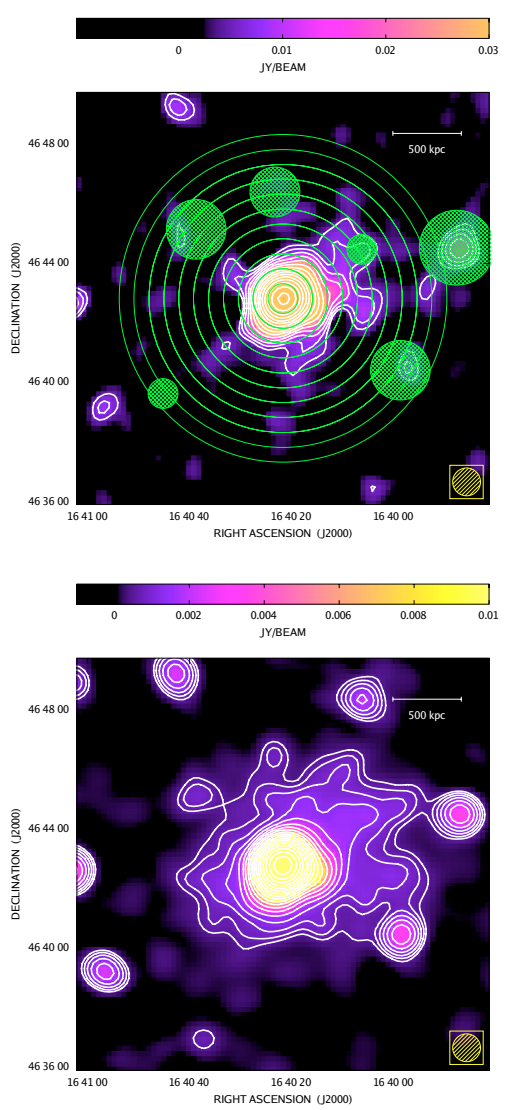

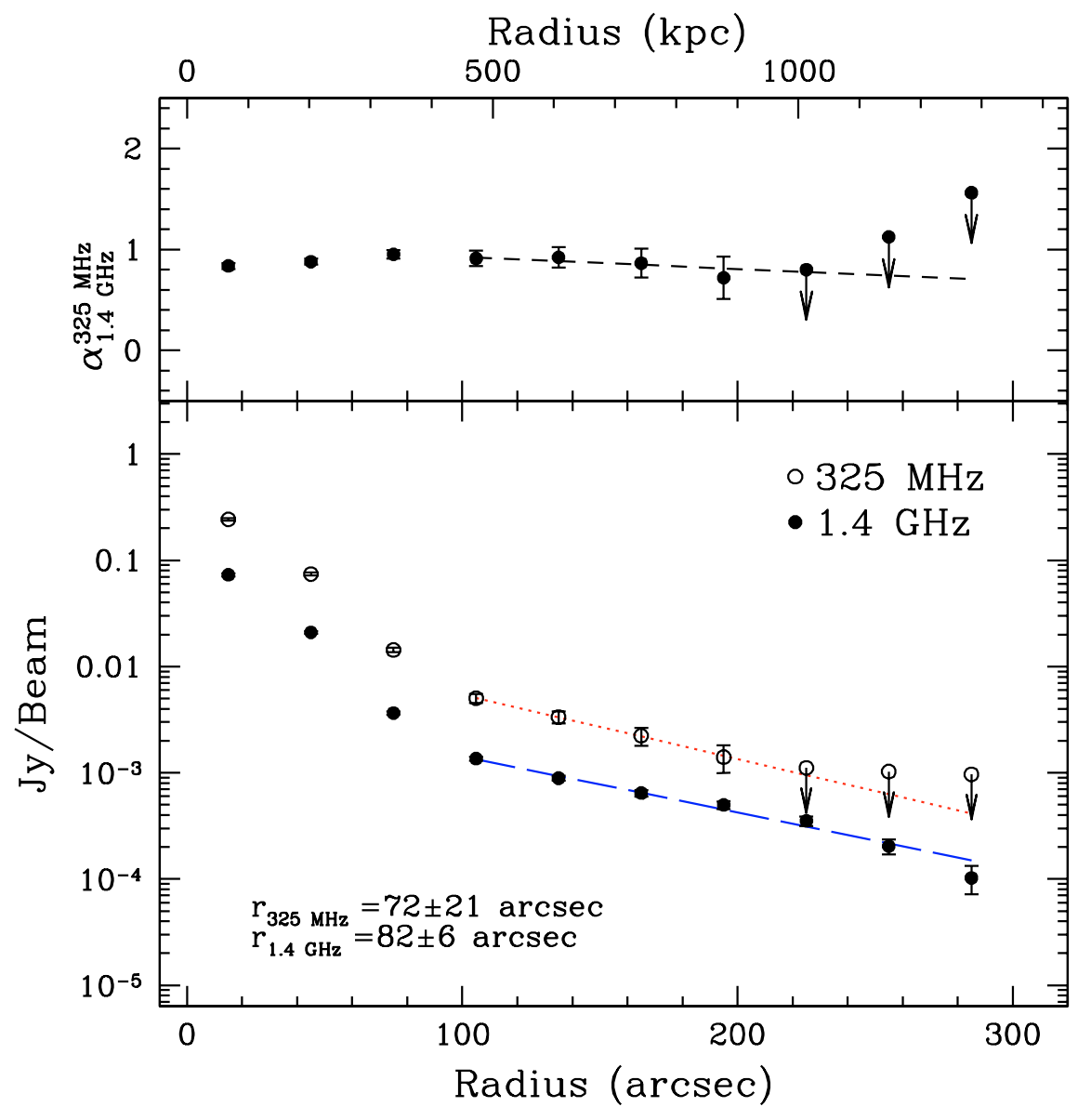

Fig. 10. A2219. Left: colors represent the images at $325 \mathrm{MHz}$ (top panel) and $1.4 \mathrm{GHz}$ (bottom panel). Contours represent the $325 \mathrm{MHz}$ and $1.4 \mathrm{GHz}$ images. Contours are spaced by $\sqrt{2}$ and start at 5.1 and $0.48 \mathrm{mJy} /$ beam at $325 \mathrm{MHz}$ and $1.4 \mathrm{GHz}$, respectively. The cross-hatched regions have been excluded from the statistics. Right: bottom panel shows the azimuthally averaged brightness profiles at $325 \mathrm{MHz}$ (open circles) and at $1.4 \mathrm{GHz}$ (solid circles). Arrows represent $3 \sigma$ upper-limits. The lines represent the fit of an exponential profile to the data (see the text). In the top panel we plot the spectral index radial profile obtained from the brightness profiles.

acceleration efficiency, the magnetic field strength in this quadrant of the cluster is constant, or slightly increasing, up to the core radius $\left(r_{\mathrm{c}} \simeq 115^{\prime \prime}\right)$ and it decreases by about a factor of two at the halo periphery.

\section{Summary}

We present new VLA images at $325 \mathrm{MHz}$ of the two clusters of galaxies A2744 and A2219, in which a wide diffuse emission has been detected at $1.4 \mathrm{GHz}$. Combining the $325 \mathrm{MHz}$ and $1.4 \mathrm{GHz}$ data, we obtained the spectral index images and the brightness radial profiles of the diffuse radio emission with a resolution of $\sim 1^{\prime}$.

A2744: the radio emission of this cluster is characterized by the presence of a radio halo and a peripheral relic. The integrated spectral index between $1.4 \mathrm{GHz}$ and $325 \mathrm{MHz}$ is $\alpha \simeq 1 \pm 0.1 \mathrm{in}$ the cluster and $\alpha \simeq 1.1 \pm 0.1$ in the relic. The azimuthally averaged spectral index in A2744 is close to a value of $\alpha \simeq 1$ up to $1 \mathrm{Mpc}$ from the cluster center. However, the spectral index image is patchy, showing regions where the spectral index is significantly different from the average. The observed spectral index variations range from a minimum of $\alpha \simeq 0.7 \pm 0.1$ to a maximum $\alpha \simeq 1.5 \pm 0.2$.

From the comparison of the spectral index image and radial profiles with the optical data from Boschin et al. (2006) and

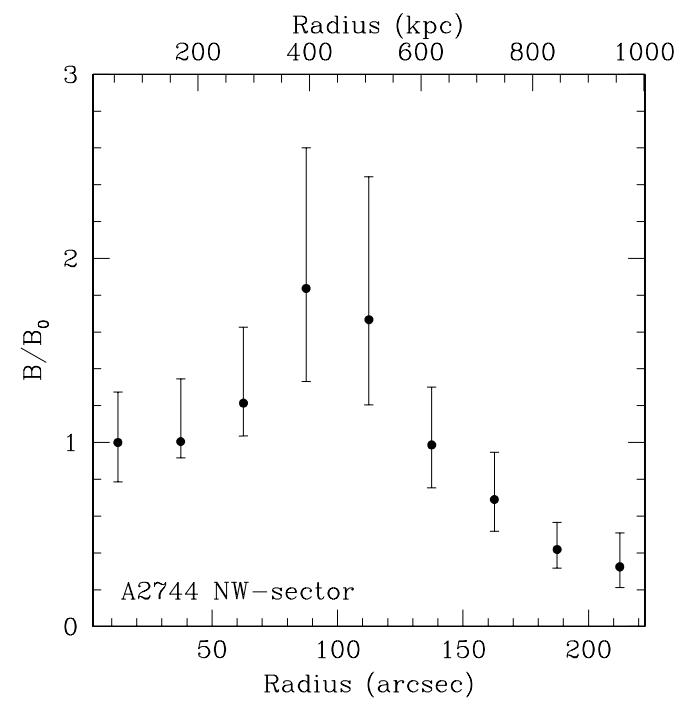

Fig. 11. Variation of the magnetic field strength in the case of constant acceleration efficiency in the NW quadrant of A2744. The magnetic field strength is normalized to its value at the cluster center.

X-ray data (Kempner \& David 2004) it appears that the southern, flat spectrum, part of the radio halo coincides in projection with 
the high-velocity group of galaxies. There is no evident correlation between the radio spectral index and the X-ray brightness substructures. However, the region of the NW group has a steep spectrum, while the the spectrum is flatter between the cluster center and the NW group. Moreover flat spectrum regions tend to have higher temperatures. This result supports the idea that a fraction of the gravitational energy, which is dissipated during major and minor mergers in heating the thermal plasma, is converted into re-acceleration of relativistic particles and amplification of the magnetic field.

A2219: the radio emission in the central regions of the cluster is dominated by the blend of discrete sources. Therefore, only the outer regions of the radio halo can be studied. The radio spectrum has an average value of $\alpha \simeq 0.8$ in the central region and a constant profile with $\alpha \simeq 1$ in the radio halo. The limited sensitivity of the $325 \mathrm{MHz}$ image does not allow us to detect all the radio halo structure seen at $1.4 \mathrm{GHz}$ and therefore no constraints on the point-to-point variations of the spectral index have been obtained for this cluster.

Acknowledgements. We are grateful to Joshua C. Kempner for the Chandra images in electronic format and Walter Boschin for the optical image. We thank Namir Kassim and Wendy Lane Peterson for advice and help and the referee Rick Perley for his criticism and helpful suggestions, which pushed us to revise the data reduction. This work was partly supported by the Italian Ministry for University and Research (MIUR) and by the Italian National Institute for Astrophysics (INAF). This research has made use of the NASA/IPAC Extragalactic Data Base (NED) which is operated by the JPL, California Institute of Technology, under contract with the National Aeronautics and Space Administration.

\section{References}

Allen, S. W. 1998, MNRAS, 296, 392

Bacchi, M., Feretti, L., Giovannini, G., et al. 2003, A\&A, 400, 465 Beck, R., \& Krause, M. 2005, AN, 326, 414

Berezinsky, V. S., Blasi, P., \& Ptuskin, V. S. 1997, ApJ, 487, 529

Blasi, P., \& Colafrancesco, S. 1999, Astroparticle Physics, 12, 169

Boschin, W., Girardi, M., Barrena, M., et al. 2004, A\&A, 416, 839

Boschin, W., Girardi, M., Spolaor, M., et al. 2006, A\&A, 449, 461
Brunetti, G., \& Blasi, P. 2005, MNRAS, 363, 1173

Brunetti, G., Setti, G., \& Comastri, A. 1997, A\&A, 325, 898

Brunetti, G., Setti, G., Feretti, L., et al. 2001, MNRAS, 320, 365

Brunetti, G., Blasi, P., Cassano, R., et al. 2004, MNRAS, 350, 1174

Carilli, C. L., \& Taylor, G. B. 2002, ARA\&A, 40, 319

Cassano, R., \& Brunetti, G. 2005, MNRAS, 357, 1313

Cho, J., \& Lazarian, A. 2006, ApJ, 638, 811

Clarke, T. E., \& Enßlin 2006, AJ, 131, 2900

Condon, J. J., Cotton, W. D., Greisen, E. W., et al. 1998, AJ, 115, 1693

Cornwell, T. J., \& Perley, R. A. 1992, A\&A, 261, 353

Dennison, B. 1980, ApJ, 239, L93

Dolag, K., \& Enßlin, T. A. 2000, A\&A, 362, 151

Enßlin, T. A., Biermann, P. L., Klein, U., \& Kohle, S. 1998, A\&A, 332, 395

Evrard, A. E., \& Gioia, I. M. 2002, Merging Processes in Galaxy Clusters, ASSL, 272253

Feretti, L., Orrù, E., Brunetti, G., et al. 2004, A\&A, 423, 111

Fujita, Y., Takizawa, M., \& Sarazin, C. L. 2003, ApJ, 584, 190

Giacintucci, S., Venturi, T., Brunetti, G., et al. 2005, A\&A, 440, 867

Giovannini, G., Feretti, L., Venturi, T., Kim, K.-T., \& Kronberg, P. P. 1993, ApJ, 406, 399

Giovannini, G., Tordi, M., \& Feretti, L. 1999, New Astron., 4, 141

Giovannini, G., \& Feretti, L. 2002, Merging Processes in Galaxy Clusters, ASSL, 272, 197

Girardi, M., \& Mezzetti, M. 2001, ApJ, 548, 79

Govoni, F., \& Feretti, L. 2004, IJMPD, 13, 1549

Govoni, F., Feretti, L., Giovannini, G., et al. 2001a, A\&A, 376, 803

Govoni, F., Enßlin, T. A., Feretti, L., \& Giovannini, G. 2001b, A\&A, 369, 441

Hwang, C.-Y. 2004, JKAS, 37, 461

Hoeft, M., \& Brüggen, M. 2007, MNRAS, tmp, 1449

Kassim, N., Perley, R. A., Erickson, W. C., \& Dwarakanath, K. S. 1993, AJ, 106, 2218

Kempner, J. C., \& David, L. P. 2004, MNRAS, 349, 385

Ohno, H., Takizawa, M., \& Shibata, S. 2002 ApJ, 577, 658

Owen, F. N., White, R. A., \& Burns, J. O. 1992, ApJS, 80, 501

Perley, R. A. 1999, Synthesis Imaging in Radio Astronomy II, ed. G. B. Taylor, C. L. Carilli, \& R. A. Perley (San Francisco: ASP), ASP Conf. Ser., 180, 383

Petrosian, V. 2001, ApJ, 557, 560

Pfrommer, C., Springel, V., Enßlin, T. A., \& Jubelgas, M. 2006, MNRAS, 367, 113

Roettiger, K., Loken, C., \& Burns, J. O. 1997, ApJS, 109, 307R

Rottgering, H. J. A., Wieringa, M. H., Hunstead, R. W., \& Ekers, R. D. 1997, MNRAS, 290, 577

Smail, I., Ellis, R. S., \& Dressler, A. 1997, ApJ, 479, 70S

Struble, M. F., \& Rood, H. J. 1999, ApJS, 125, 35

Tribble, P. C. 1993, MNRAS, 263, 31 